\title{
Molecular Biology of Breast Cancer in the Horn of Africa: Case Series-A Pilot Study of Breast Cancer from Eritrea
}

\author{
Asmerom Tesfamariam ${ }^{1}$ and Indrojit Roy ${ }^{2}$ \\ ${ }^{1}$ Orotta School of Medicine and Dentistry, Department of Pathology, Asmara, Eritrea \\ ${ }^{2}$ St. Mary's Hospital, McGill University, Department of Pathology, Montreal, QC, Canada H3T 1M5
}

Correspondence should be addressed to Asmerom Tesfamariam; asmeromp@gmail.com

Received 12 March 2013; Accepted 4 May 2013

Academic Editors: E. E. Akang, A. Gocht, and A. Stringer

Copyright (C) 2013 A. Tesfamariam and I. Roy. This is an open access article distributed under the Creative Commons Attribution License, which permits unrestricted use, distribution, and reproduction in any medium, provided the original work is properly cited.

Background. Recently, gene expression profiling and its surrogate immunohistochemistry (IHC) markers classified breast cancer into four distinct molecular subtypes, which have different prognoses, targeted therapies, and/or clinical outcomes. Objective. To conduct a preliminary study, to correlate the clinical pathological profiles and taxonomy of molecular subtypes of breast cancer in Eritrea, in the Horn of Africa. Design. Review of pathology reports from Jan. 1 to Nov. 30, 2009, provided 22 cases of microscopically confirmed invasive breast carcinoma that were evaluable for histology and IHC (ER, PR, HER2, and Cytokeratin 5/6). Result. Twenty patients were female and most of them $(68 \%)$ were under 50 years at presentation. $90 \%$ were invasive invasive carcinoma of no special type and were histological grade 3. The molecular subtypes were luminal A (55\%), luminal B (5\%), HER2 (5\%), basal-like (10\%), and unclassified (25\%). Triple negative carcinoma (basal-like and unclassified combined) was 35\%, mostly (71\%) in women under 50 years with grade 3 tumours. Conclusion. Breast carcinoma in Eritrean women presents at a younger age and with a high histologic grade. The two predominant molecular subtypes are luminal A and triple negative. Determining the molecular subtype using surrogate IHC markers has important treatment and prognostic implications for Eritrean women with breast cancer.

\section{Introduction}

Among women, breast cancer is the leading cause of cancerrelated death in the world [1]. The lowest incidence is in Africa; however, the incidence has recently been increasing and is accompanied by increased mortality [1, 2]. Breast cancer in African and African-American women is characterized by late presentation, younger age, advanced stage, higher grade, more negative hormonal receptor status, and poorer prognosis, when compared to Caucasian American and western European women [3-6]. Although the causes for these disparities may include socioeconomic status, access to screening, and differences in treatment decisions, the intrinsic biology of the disease itself may play a role in the different outcomes [7-9].

Today, breast cancer is considered to be a heterogeneous disease, consisting of different molecular subtypes which correlate with disease outcomes independent of other factors. Gene expression microarrays have identified distinct breast cancer molecular subtypes, including two types of estrogen receptor (ER) negative tumors: basal-like and human epidermal growth receptor (HER2) enriched, and two types of ERpositive tumors: luminal A and luminal B [10, 11]. A simpler method employs immunohistochemistry (IHC) to identify these molecular subtypes, using antibodies to ER, progesterone receptor (PR), HER2, and myoepithelial markers such as cytokeratin (CK) 5/6. Although gene microarray analysis is more accurate, it is not routinely used in clinical practice, as it is more expensive.

Using these surrogate IHC markers, breast cancer is classified into luminal A (ER+ and/or PR+, HER2-), luminal $\mathrm{B}(\mathrm{ER}+$ and/or PR+, HER2+), HER2 type (ER-, PR-, and HER2+), basal-like (ER-, PR-, HER2-, and CK 5/6 positive), and unclassified (negative for all markers) [12, 13]. 
The Carolina breast cancer study demonstrated a distribution of $51 \%, 16 \%, 7 \%, 20 \%$, and $6 \%$ for luminal A, luminal B, HER2 type, basal-like, and unclassified, respectively [12]. By contrast, a study of indigenous West African women found that the proportions of luminal A, luminal B, HER2 type, basal-like, and unclassified tumors were 27\%, 2\%, 15\%, $27 \%$, and $28 \%$, respectively [14]. Another study from Nigeria found that $77.6 \%$ were luminal type $A, 2.6 \%$ luminal type B, $15.8 \%$ basal-like, and the remaining 4.0\% HER2 type [15] A study from North Africa (Egypt) reported 44\% luminal A, 26.6\% luminal B, 11.8\% HER2 type, 11.3\% basal-like, and $7.9 \%$ unclassified [16], while a study from Sudan found a high proportion of ER-and PR-positive tumours [17] and lower basal-like (10\%) phenotypes [18]. This last study did not assess the HER2 type of breast carcinoma. The published literature therefore has not demonstrated a consistent pattern of breast cancer biology within the continent of Africa.

In general, patients with the luminal A subtype have the best prognosis, and patients with luminal B have a relatively poorer prognosis $[12,19]$. Both the basal-like and HER2 subtypes have the poorest prognosis. However, more recently, with HER2 targeting agents, outcomes for the HER2 and HER2-positive luminal cancers have markedly improved. The basal-like triple negative breast cancer is the most aggressive and appears to be more common in premenopausal AfricanAmerican women as compared to postmenopausal AfricanAmerican and non-African women (39\%, 14\%, and 16\% resp.). In contrast, luminal A tumors with good prognosis are less common in African-American women [12, 20]. Studies of breast cancer in African women show they occur at a younger age, express hormone receptors less frequently and more often are triple negative $[21,22]$.

The aforementioned studies suggest that molecular subtypes differ in geographic distribution, race, age, prognosis, and the therapeutic targets they express. It may be that breast cancers in different regions of Africa have distinct biological behavior. These recent studies with IHC are proxies for the potentially unique biologies of breast cancer in different regions of Africa, and more detailed investigations, such as gene microarray expression profiling and proteomics are needed to advance our understanding.

In the Horn of Africa, including Eritrea, the prevalence of the molecular subtypes and their correlation with clinicalpathological features has not been reported. The objective of this study was to define these parameters, using surrogate IHC analysis and, thus, to provide evidence-based data for future guidelines on breast carcinoma management. This is the first such case series pilot study in this region of Africa.

\section{Materials and Methods}

2.1. Study Design and Sample Collection. With the approval of the Research Ethics Committee of the Orotta School of Medicine and Dentistry, bound paper copies of all pathology reports from January 1, 2009, to November 30, 2009 were screened for histologically diagnosed invasive breast cancer, and 33 patients were identified. 80 formalin-fixed and paraffin-embedded (FFPE) blocks from 30 of these cases were retrieved from the archives of the Pathology Department, the National Health Laboratory (Asmara), Eritrea. Blocks from 3 cases could not be located. Haematoxylin and Eosin $(\mathrm{H}+$ E) slides were prepared from each block, and invasive breast carcinoma with adequate tissue for study was identified in 22 cases. The remaining 8 cases were excluded either because the selected blocks did not contain invasive carcinoma (2 cases with DCIS only and 4 cases with no evidence of carcinoma) or because the tissue preservation was considered too poor for reliable histological or IHC interpretation (2 cases). Age, gender, tumour size, and lymph node status of each patient were obtained from the pathology reports. Slides were cut and stained at St. Mary's Hospital, Montreal, Quebec, Canada, and interpreted by one of the authors.

2.2. Immunohistochemistry. The histologic subtype of invasive breast carcinoma and Nottingham histologic grade were defined using the routine $(\mathrm{H}+\mathrm{E})$ morphological evaluation. Immunostaining was done for estrogen receptor (ER), progesterone receptor (PR), human epidermal growth factor 2 (HER2/neu), and CK5/6, using a Ventana Benchmark immunostainer (Tucson, Arizona), using the manufacturer's antibodies (clones 6F11 for anti-ER, 1A6 for anti-PR, 4B5 for HER2/neu, and D5/16B4 for CK5/6). ER and PR were considered positive if $1 \%$ or more of the nuclei of invasive carcinoma cells were stained. HER2/neu stains were assessed as $0,1+, 2+$, and $3+$ using the College of American Pathologists guidelines [23]. Specifically, 3+ represented uniform intense membrane staining of more than $30 \%$ of the tumour cells, and was considered positive for HER2/neu overexpression. A lesser degree of staining was considered negative. Fluorescence in situ hybridization for HER2/neu gene amplification was not performed. Cytokeratin 5/6 was scored positive if any (weak or strong) cytoplasmic and/or membranous invasive carcinoma cell staining was observed [24].

Color development and background staining was visualized using the 3,3-diaminobenzadine chromagen and haematoxylin counter stain, respectively. Appropriate negative controls for immunostaining were prepared by eliminating the primary antibody step.

As previously defined [12], the combination of IHC markers used to define breast cancer molecular subtypes in this study was as follows luminal A (ER positive and/or PR positive, Her2 negative), luminal B (ER positive and/or PR positive, Her2 positive), Her2 type (ER negative, PR negative, Her2 positive), basal-like (ER negative, $\mathrm{PR}$ negative, Her2 negative, and CK5/6 positive), and unclassified (ER, PR, Her2, and CK5/6 negative). By this definition, the "triple negative" (ER, PR, and Her2 negative) tumours incorporate both the basal-like and the unclassified categories.

2.3. Data Analysis. Data analysis was done using SPSS version 17. Tumor behaviors were compared across the breast cancer subtypes using the Chi-square $\left(\chi^{2}\right)$ test for categorical variables. One-way analysis of variance was conducted to compare the difference in age at diagnosis between breast cancer subtypes. Kruskal-Wallis tests were used to compare tumor size, histologic grade between subtypes. Fisher's exact 
TABLE 1: Summary of the clinical pathological characteristics of molecular subtypes of breast cancer in Eritrea.

\begin{tabular}{|c|c|c|c|c|c|c|c|}
\hline \multirow{3}{*}{ Characteristics } & \multicolumn{7}{|c|}{ Molecular subtypes of breast cancer } \\
\hline & $\begin{array}{c}\text { All cases } \\
N=22(100)\end{array}$ & $\begin{array}{c}\text { Luminal A } \\
n=13(59)\end{array}$ & $\begin{array}{l}\text { Luminal B } \\
n=1(4.5)\end{array}$ & $\begin{array}{l}\text { HER2 type } \\
n=1(4.5)\end{array}$ & $\begin{array}{c}\text { Basal-like } \\
n=2(9.1)\end{array}$ & $\begin{array}{l}\text { Unclassified } \\
n=5(22.7)\end{array}$ & $P$ value \\
\hline & $N(\%)$ & $n(\%)$ & $n(\%)$ & $n(\%)$ & $n(\%)$ & $n(\%)$ & \\
\hline \multicolumn{8}{|l|}{ Sex } \\
\hline Male & $2(9.1)$ & $2(18.2)$ & $0(0)$ & $0(0)$ & $0(0)$ & $0(0)$ & 0.70 \\
\hline Female & $20(90.9)$ & $11(84.6)$ & $1(100)$ & $1(100)$ & $2(100)$ & $5(100)$ & \\
\hline \multicolumn{8}{|l|}{ Age in years } \\
\hline$<50$ & $13(63.6)$ & $7(63.6)$ & 1 & 0 & 2 & $4(80)$ & 0.26 \\
\hline$\geq 50$ (age unknown in one patient) & $8(36.4)$ & $4(36.4)$ & 0 & 2 & 0 & $2(20)$ & \\
\hline Mean (SD) & $47.7(12.9)$ & & & & & & \\
\hline \multicolumn{8}{|l|}{ Histologic type } \\
\hline IC (NST) & $20(91)$ & $10(90.1)$ & 1 & 2 & 1 & 6 & 0.18 \\
\hline Colloid & $1(4.5)$ & $1(9.9)$ & 0 & 0 & 0 & 0 & \\
\hline Metaplastic breast carcinoma (SCC) & $1(4.5)$ & 0 & 0 & 0 & 1 & 0 & \\
\hline \multicolumn{8}{|l|}{ Nottingham grade } \\
\hline I & $2(9.1)$ & $2(18.2)$ & 0 & 0 & 0 & 0 & 0.031 \\
\hline II & $7(31.8)$ & $6(54.5)$ & 0 & 0 & 0 & $1(16.7)$ & \\
\hline III & $13(59.1)$ & $3(27.3)$ & 1 & 2 & 2 & $5(83.3)$ & \\
\hline \multicolumn{8}{|l|}{$\mathrm{LN}^{*}$ metastasis } \\
\hline Positive & $11(50)$ & $6(54.5)$ & 1 & 1 & $1(50)$ & $2(33.3)$ & 0.44 \\
\hline Negative & $8(36.4)$ & $5(45.5)$ & 0 & 0 & 0 & $2(33.3)$ & \\
\hline NA & $3(13.6)$ & 0 & 0 & 0 & $1(50)$ & $2(33.3)$ & \\
\hline \multicolumn{8}{|l|}{ Tumor size } \\
\hline$\leq 2 \mathrm{~cm}$ & $1(4.5)$ & $1(9.1)$ & 0 & 0 & 0 & 0 & 0.84 \\
\hline $2.1-5 \mathrm{~cm}$ & $12(54.6)$ & $6(54.5)$ & 1 & 1 & 0 & 4 & \\
\hline$>5 \mathrm{~cm}$ & $7(31.8)$ & $4(36.4)$ & 0 & 1 & 1 & 1 & \\
\hline NA & $2(9.1)$ & 0 & 0 & 0 & 1 & 1 & \\
\hline \multicolumn{8}{|l|}{$\mathrm{LVI}^{* *}$} \\
\hline Positive & $3(13.6)$ & $1(9.9)$ & 1 & 0 & $1(50)$ & 0 & 0.04 \\
\hline Negative & $19(86.4)$ & $10(90.1)$ & 0 & 2 & $1(50)$ & 6 & \\
\hline \multicolumn{8}{|l|}{$\mathrm{ITN}^{+}$} \\
\hline Positive & $10(45.4)$ & $4(36.4)$ & 1 & 2 & 0 & $3(50)$ & 0.11 \\
\hline Negative & $12(54.6)$ & $7(63.6)$ & 0 & 0 & 2 & $3(50)$ & \\
\hline
\end{tabular}

$\mathrm{ITN}^{+}$: invasive tumour necrosis, $\mathrm{LVI}^{* *}$ : lymphatic vessel invasion, $\mathrm{LN}^{*}$ : lymph node, IC (NST): invasive carcinoma of no special type, NA: Nonavailable, $P$ : probability, SD: standard deviation, SCC: squamous cell carcinoma.

tests was used to examine the relationship between categorical pathologic characteristics and subtypes which have less than 5 categorical cells. All $P$ values were based on 2-tailed tests of significance where $P<0.05$ is considered statistically significant.

\section{Results}

A total of 22 cases were evaluated for biomarkers using immunohistochemistry in which two-thirds (64\%) were ER positive. The age ranged from 26 to 76 years and the mean age was 47.7 years with standard error of the mean 2.7. About $55 \%$ had tumor size between 2.1 to $5 \mathrm{~cm}$ and $45 \%$ had microscopic invasive tumour necrosis. Table 1 summarizes the clinical pathological characteristics of molecular subtypes of breast cancer in our series of patients. Both male patients were over 50 years of age, one with a grade 1 and the other with a grade 3 tumour, and both had Luminal A breast carcinoma.

With respect to breast carcinoma in females, two-thirds of the patients, whose age was known, were under 50 years $(68 \%$, 


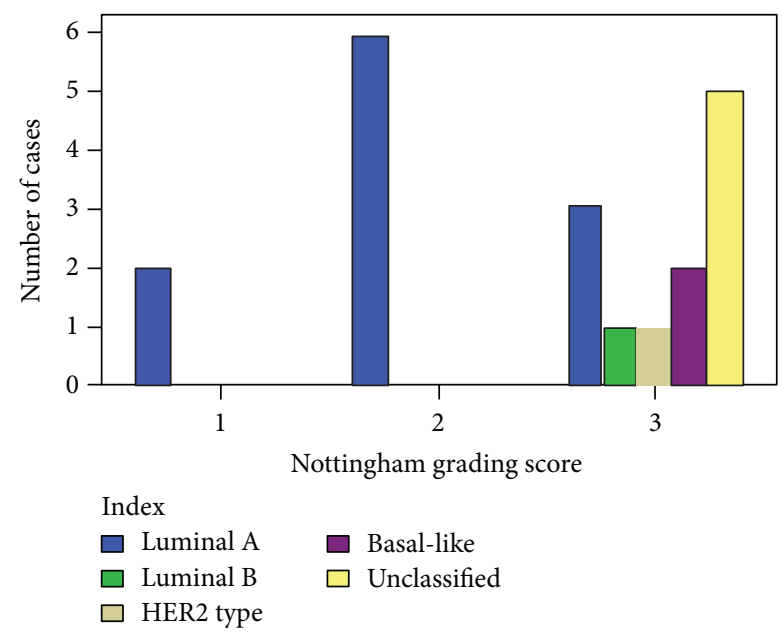

FIGURE 1: Distribution of molecular subtypes of female breast cancer according to Nottingham grading score.

13/19). Most (90\%) of the invasive carcinomas were invasive carcinoma of no special type (IC NST). One was a colloid (mucinous) carcinoma and one was a metaplastic (squamous cell) carcinoma of the breast (SCC). $60 \%$ (12/20 patients) were Nottingham grade III. 40\% (8/20 patients) were ER negative and $60 \%(12 / 20$ patients) were ER positive. The molecular subtypes of breast carcinoma in females were as follows: luminal A $55 \%$ (11/20 patients, one of the cases is illustrated in Figure 2), luminal B 5\% (1/20 patients), Her2 type 5\% (1/20 patients, illustrated in Figure 3$)$, basal-like 10\% (2/20 patients as illustrated in Figure 4), and unclassified 25\% (5/20 patients). Thus, "triple negative" tumours (basal-like and unclassified) accounted for $35 \%$ of patients (7/20 patients). Most of these women with "triple negative" tumours were under 50 years (71\%, 5/7 patients) and had grade 3 tumours (86\%, 6/7 patients).

The distribution of molecular subtypes was independent of tumour size, lymph node metastasis and invasive tumour necrosis. There was significant association between molecular subtype and grade and lymphatic invasion $(P=0.03$ and $0.04)$, respectively.

Luminal A tumours were mostly Nottingham grade 1 or $2(64 \%, 7 / 11)$, whereas most of the other molecular intrinsic subtypes were grade $3(8 / 9,89 \%)$, as shown in Figure 1.

\section{Discussion}

This case series study indicates that the majority of Eritrean women presenting with breast cancer are less than 50 years of age. Invasive carcinoma (NST) is the most common histologic type, and the majority are Nottingham grade 3. Similar findings have been reported in native African $[15,17$, 21, 25] and African-American women [12]. Though our study is not a large case series, there is an association between high tumor grade and the aggressive intrinsic molecular subtypes, which is consistent with the findings in many other studies $[12,14,16]$.
In this study, the overall proportion of ER-positive tumors in females was $60 \%$. Even though our sample size is small, our findings are very similar to those from Uganda [25] and in African-American women [12] but slightly lower than those from those Egypt [16] and central Sudan [17], although much higher than West Africa (Nigeria and Senegal) [14]. In our case series, about half of the cases were luminal A which has a good prognosis, a superior response to hormonal therapy, and a higher survival rate. This finding is consistent with the population-based Carolina Breast Cancer Study [12] as well as North Africa and Egypt [16, 24], but quite different from the study in Nigeria and Senegal $[14,15]$. Luminal B (with a poorer prognosis and less responsive to hormonal therapy as compared to luminal type A) is less frequent when compared to the studies from Carolina [12] and Egypt [16] but very similar to the West African Study [14]. The proportion of Her2 type in our study was $5 \%$, similar to the findings from Uganda [25], Egypt, [16] and the population-based Carolina Breast Cancer study [12], but less than the $19 \%$ estimate by the population based study from United States in premenopausal women [26]. The percentage of the basal-like subtype in our series is $10 \%$ which is consistent with the findings from central Sudan $[17,18]$ and Egypt $[16]$ but lower than the 27\% found in the Nigerian/Senegalese study [14]. However, in that study, the basal-like subtype was defined as being either CK5/6 or EGFR positive. It is currently accepted that using CK5/6 alone may miss about half of the basal-like subtype, and it is likely that we have underestimated the proportion of this subtype. Therefore, it is possible that approximately $20 \%$ of our cases may be basal-like, a figure which is much closer to the Nigerian/Senegalese studies [14,27]. The broader category of triple negative breast cancer (TNBC), defined by a negative ER and PR, and lack of HER2 overexpression represent 35\% of our cases, which is consistent with Ugandan [25] and other studies [13, 21], but lower than the $49 \%$ reported from Nigeria [27].

We note that the prevalence of the molecular subtypes of breast carcinoma appears to have variation in geographical distribution. The reasons for these differences could be technical, such as the quality of tissue fixation and processing, varying staining techniques, and different criteria in scoring and reporting. It is also possible that the molecular phenotypes of African breast cancer is biologically diverse, and the result of regional population genetic differences, as well as environmental factors. For example, the prevalence of BRCA1/2 mutations is not significantly higher in African-American women as compared to CaucasianAmerican women, in spite of the finding that both the basallike and the "triple negative" subtypes are more frequent [28], suggesting that there are probably other genetic pathways for this molecular subtype. The potential contribution of environmental causes is supported by the finding that for "triple negative" breast cancer in African-American women, the risk is increased with parity and younger age at first term full-term pregnancy, whereas there is a reduced risk associated with a longer duration of breastfeeding, increasing number of children breast-fed, and increasing number of months of breast-feeding. This observation was not seen among those diagnosed with luminal A breast cancer [29]. 


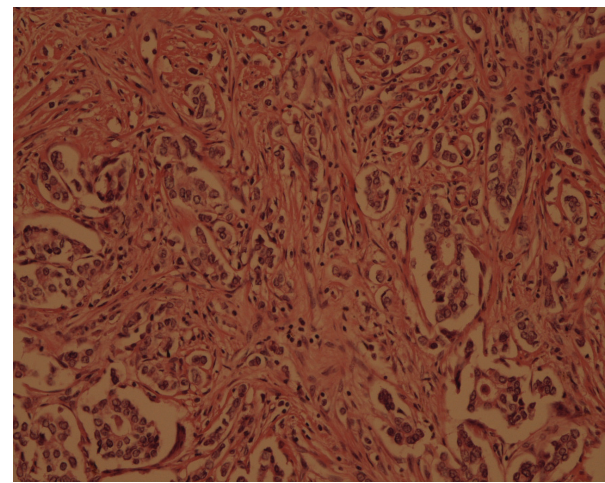

(a)

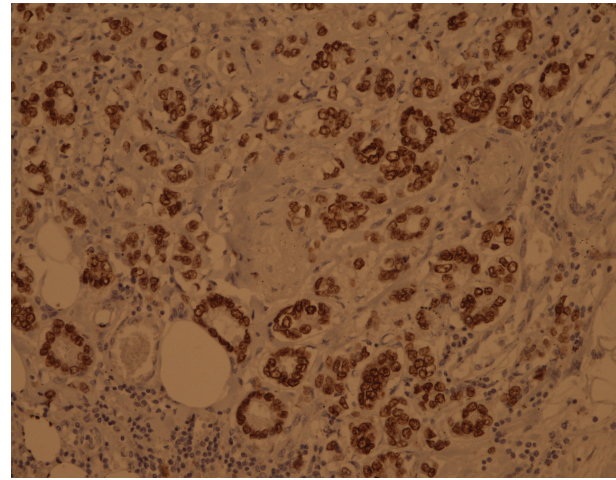

(b)

Figure 2: (a) and (b) the H + E and ER IHC of luminal A breast carcinoma. (a) H + E stain, Nottingham grade 1, 200x magnification. (b) IHC for ER positive. Moderate to strong nuclear stain in 90\% of invasive carcinoma, 200x magnification. PR was positive and both the Her2 and CK5/6 were negative (not illustrated here).

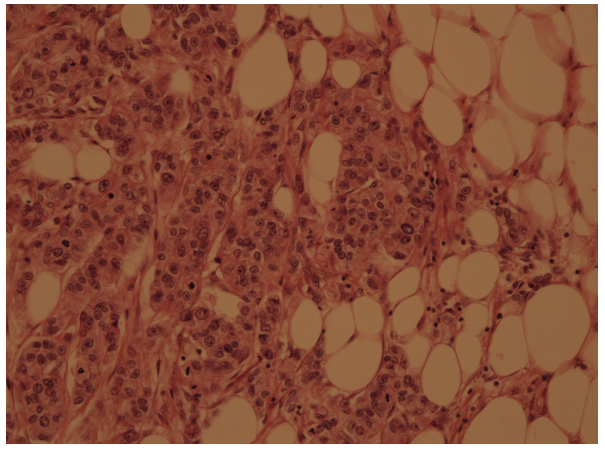

(a)

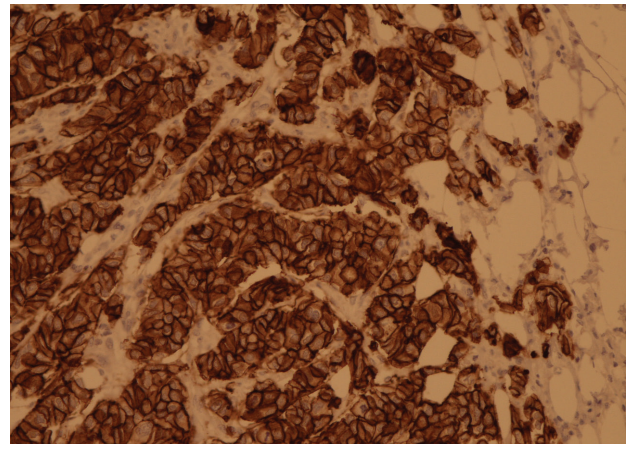

(b)

Figure 3: (a) and (b), H + E and HER 2 IHC of HER2 type of invasive ductal carcinoma. (a) H + E stain. Nottingham grade 3; 200x magnification. (b) IHC for Her2/neu. Positive for overexpression (uniform intense membrane staining of more than $30 \%$ of the tumor cells). 200x magnification. The ER, PR, and CK5/6 were negative (not illustrated here).

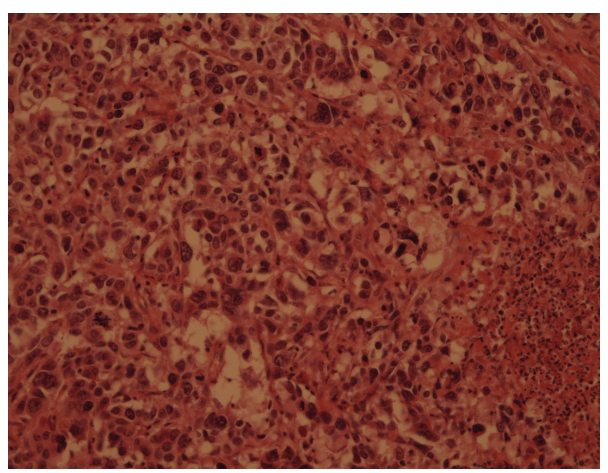

(a)

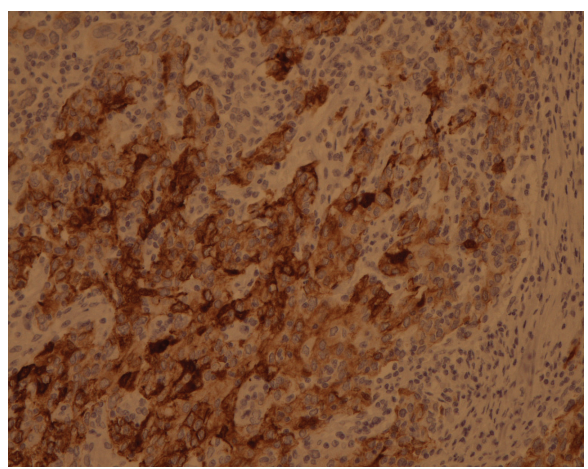

(b)

Figure 4: (a) and (b), H + E and CK 5/6 positive basal like invasive breast cancer (a) H + E stain. Nottingham grade 3. Note invasive tumour necrosis on the right. 200x magnification. (b) IHC for CK5/6 positive. Moderate to strong cytoplasmic staining of invasive carcinoma, 200x magnification. The ER, PR, and Her2 were negative (not illustrated here). 
Knowledge of regional variation in molecular subtypes may inform therapy, when biomarker evaluation is not available. However, if biomarkers are available, clinicians should continue to manage patients based on that status, since there is significant individual variation. In Eritrea, determination of routine hormone receptor status and biomarker analysis has not yet been established and the only available means of treatment is surgery [30]. A lot of efforts are waiting for the young nation to implement the standard guideline of breast cancer management, which is currently used in most advanced setting.

There are some limitations to this study. First, it has a small number of cases. Second, it depends on the accuracy of archived pathology records. Third, although there were 33 histologically confirmed breast cancer cases identified in the pathology records for the time period assessed, only 22 were used in this study, primarily because the FFPE blocks with invasive carcinoma could not be located. It is possible that this gap could significantly have impacted our findings. The tissue processing may not have been uniform and therefore may have affected the immunostain results (prolonged formalin fixation is thought to affect antigen preservation). The data may not apply to all Eritrean women because the study was carried out in small case series. In addition, the lack of consistent data regarding tumor size and stage might bias the results.

\section{Conclusion}

In summary, this pilot case series suggests that the majority of breast cancer in Eritrea lies at the two ends of the molecular spectrum, that is, luminal A (55\% of patients), sensitive to hormonal therapy and with a good prognosis, and "triple negative" (35\% of patients, basal-like, and unclassified combined), not hormonally sensitive and with a poor prognosis, for which new treatments are needed. These findings suggest that biomarker analyses in the pathology laboratory are critical for the effective management of breast cancer in Eritrean women.

\section{Acknowledgment}

The authors would like to express gratitude to the Eritrean Ministry of Health, especially the National health Laboratory, pathology department for its full cooperation in obtaining the valuable data. Furthermore, our appreciation goes to Profesor Andemariam Gebremichael (Dean of the Orotta School of Medicine and Dentistry), Profesor Jacob Mufunda, and Dr. Godwins for their precious advice in writing this paper.

\section{References}

[1] J. Ferlay, H. R. Shin, F. Bray, D. Forman, C. Mathers, and D. M. Parkin, "Estimates of worldwide burden of cancer in 2008: GLOBOCAN 2008," International Journal of Cancer, vol. 127, no. 12, pp. 2893-2917, 2010.

[2] G. N. Hortobagyi, J. de la Garza Salazar, K. Pritchard et al., "The global breast cancer burden: variations in epidemiology and survival," Clinical Breast Cancer, vol. 6, no. 5, pp. 391-401, 2005.

[3] J. W. Eloy, H. A. Hill, V. W. Chen et al., "Racial differences in survival from breast cancer: results of the National Cancer Institute Black/White Cancer Survival Study," Journal of the American Medical Association, vol. 272, no. 12, pp. 947-954, 1994.

[4] R. T. Chlebowski, Z. Chen, G. L. Anderson et al., "Ethnicity and breast cancer: factors influencing differences in incidence and outcome," Journal of the National Cancer Institute, vol. 97, no. 6, pp. 439-448, 2005.

[5] K. C. Chu and W. F. Anderson, "Rates for breast cancer characteristics by estrogen and progesterone receptor status in the major racial/ethnic groups," Breast Cancer Research and Treatment, vol. 74, no. 3, pp. 199-211, 2002.

[6] R. L. Bowen, S. W. Duffy, D. A. Ryan, I. R. Hart, and J. L. Jones, "Early onset of breast cancer in a group of British black women," British Journal of Cancer, vol. 98, no. 2, pp. 277-281, 2008.

[7] R. Smith-Bindman, D. L. Miglioretti, N. Lurie et al., "Does utilization of screening mammography explain racial and ethnic differences in breast cancer?" Annals of Internal Medicine, vol. 144, no. 8, pp. 541-553, 2006.

[8] L. A. Newman, K. A. Griffith, I. Jatoi, M. S. Simon, J. P. Crowe, and G. A. Colditz, "Meta-analysis of survival in African American and white American patients with breast cancer: ethnicity compared with socioeconomic status," Journal of Clinical Oncology, vol. 24, no. 9, pp. 1342-1349, 2006.

[9] C. M. Perou, T. Sørile, M. B. Eisen et al., "Molecular portraits of human breast tumours," Nature, vol. 406, no. 6797, pp. 747-752, 2000.

[10] T. Sørlie, C. M. Perou, R. Tibshirani et al., "Gene expression patterns of breast carcinomas distinguish tumor subclasses with clinical implications," Proceedings of the National Academy of Sciences of the United States of America, vol. 98, no. 19, pp. 1086910874, 2001.

[11] T. Sørlie, R. Tibshirani, J. Parker et al., "Repeated observation of breast tumor subtypes in independent gene expression data sets," Proceedings of the National Academy of Sciences of the United States of America, vol. 100, no. 14, pp. 8418-8423, 2003.

[12] L. A. Carey, C. M. Perou, C. A. Livasy et al., "Race, breast cancer subtypes, and survival in the Carolina Breast Cancer Study," Journal of the American Medical Association, vol. 295, no. 21, pp. 2492-2502, 2006.

[13] T. O. Nielsen, F. D. Hsu, K. Jensen et al., "Immunohistochemical and clinical characterization of the basal-like subtype of invasive breast carcinoma," Clinical Cancer Research, vol. 10, pp. 5367-5374, 2004.

[14] D. Huo, F. Ikpatt, A. Khramtsov et al., "Population differences in breast cancer: survey in indigenous african women reveals over-representation of triple-negative breast cancer," Journal of Clinical Oncology, vol. 27, no. 27, pp. 4515-4521, 2009.

[15] C. A. Adebamowo, A. Famooto, T. O. Ogundiran, T. Aniagwu, C. Nkwodimmah, and E. E. Akang, "Immunohistochemical and molecular subtypes of breast cancer in Nigeria," Breast Cancer Research and Treatment, vol. 110, no. 1, pp. 183-188, 2008.

[16] B. S. Coya Tapia, A. Elia Ishak, S. Gaber et al., "Molecular subtype analysis determines the association of advanced breast cancer in Egypt with favorable biology," Journal of BMC Womens' Health, vol. 44, no. 11, pp. 1472-11478, 2011.

[17] K. D. Awadelkarim, C. Arizzi, E. O. M. Elamin et al., "Pathological, clinical and prognostic characteristics of breast cancer 
in Central Sudan versus Northern Italy: implications for breast cancer in Africa," Histopathology, vol. 52, no. 4, pp. 445-456, 2008.

[18] K. D. Awadelkarim, C. Arizzi, E. O. M. Elamin et al., "Basallike phenotype in a breast carcinoma case series from Sudan: prevalence and clinical/pathological correlations," Pathology Research International, vol. 2011, Article ID 806831, 10 pages, 2011.

[19] C. Fan, D. S. Oh, L. Wessels et al., "Concordance among geneexpression-based predictors for breast cancer," The New England Journal of Medicine, vol. 355, no. 6, pp. 560-569, 2006.

[20] K. M. O’Brien, S. R. Cole, C. K. Tse et al., "Intrinsic breast tumor subtypes, race, and long-term survival in the carolina breast cancer study," Clinical Cancer Research, vol. 16, no. 24, pp. 61006110, 2010.

[21] P. A. Bird, A. G. Hill, and N. Houssami, "Poor hormone receptor expression in East African breast cancer: evidence of a biologically different disease?" Annals of Surgical Oncology, vol. 15, no. 7, pp. 1983-1988, 2008.

[22] R. Bhikoo, S. Srinivasa, T. C. Yu, D. Moss, and A. G. Hill, "Systematic review of breast cancer biology in developing countries (part 1): Africa, the Middle East, Eastern Europe, Mexico, the Caribbean and South America," Cancers, vol. 3, no. 2, pp. 2358-2381, 2011.

[23] A. C. Wolff, M. E. Hammond, J. N. Schwartz et al., "American Society of Clinical Oncology/College of American Pathologists guideline recommendations for human epidermal growth factor receptor 2 testing in breast cancer," Journal of Clinical Oncology, vol. 25, pp. 118-145, 2007.

[24] A. K. El-Hawary, A. S. Abbas, A. A. Elsayed, and K. R. Zalata, "Molecular subtypes of breast carcinoma in Egyptian women: clinicopathological features," Pathology, vol. 208, no. 7, pp. 382386, 2012.

[25] I. Roy and E. Othieno, "Breast carcinoma in Uganda: microscopic study and receptor profile of 45 cases," Archives of Pathology and Laboratory Medicine, vol. 135, no. 2, pp. 194-199, 2011.

[26] K. A. Cronin, L. C. Harlan, K. W. Dodd, J. S. Abrams, and R. Ballard-Barbash, "Population-based estimate of the prevalence of HER-2 positive breast cancer tumors for early stage patients in the US," Cancer Investigation, vol. 28, no. 9, pp. 963-968, 2010.

[27] K. A. Adeniji, D. Huo, A. Khramtsov, C. Zhang, and O. I. Olopade, "Molecular profiles of breast cancer in Ilorin, Nigeria," Journal of Clinical Oncology, vol. 28, no. 15, supplement, 2010, abstract no.1602.

[28] K. E. Malone, J. R. Daling, D. R. Doody et al., "Prevalence and predictors of BRCA1 and BRCA2 mutations in a populationbased study of breast cancer in White and Black American women ages 35 to 64 years," Cancer Research, vol. 66, no. 16, pp. 8297-8308, 2006.

[29] C. K. Anders and L. A. Carey, "Biology, metastatic patterns, and treatment of patients with triple-negative breast cancer," Clinical Breast Cancer, vol. 9, no. 2, pp. S73-S81, 2009.

[30] A. Tesfamariam, A. Gebremichael, and J. Mufunda, "Overview of breast cancer clinicopathological presentation,gravity, and challenge in Eritrea," South African Medical Journal. In press. 


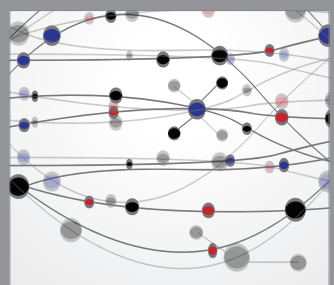

The Scientific World Journal
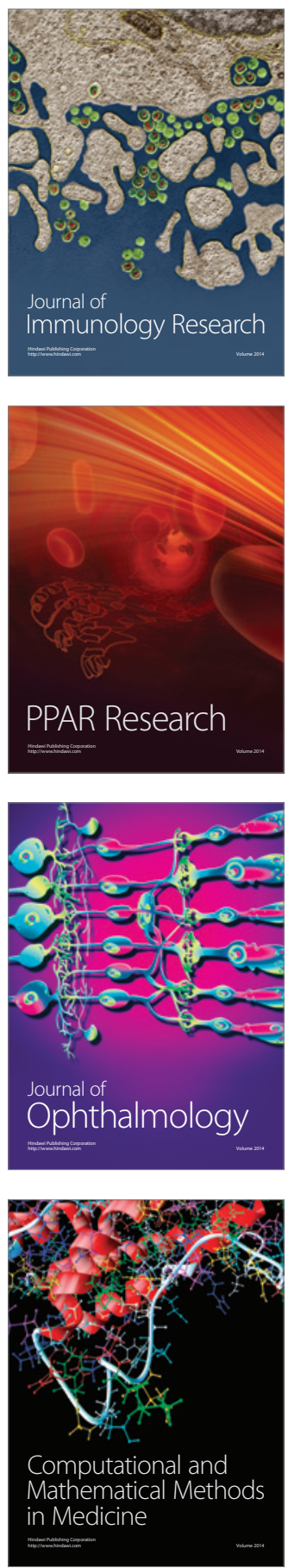

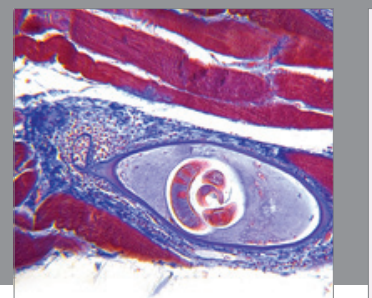

Gastroenterology

Research and Practice
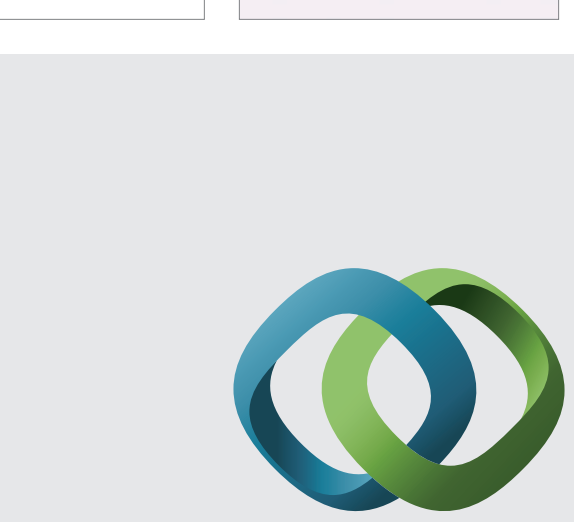

\section{Hindawi}

Submit your manuscripts at

http://www.hindawi.com
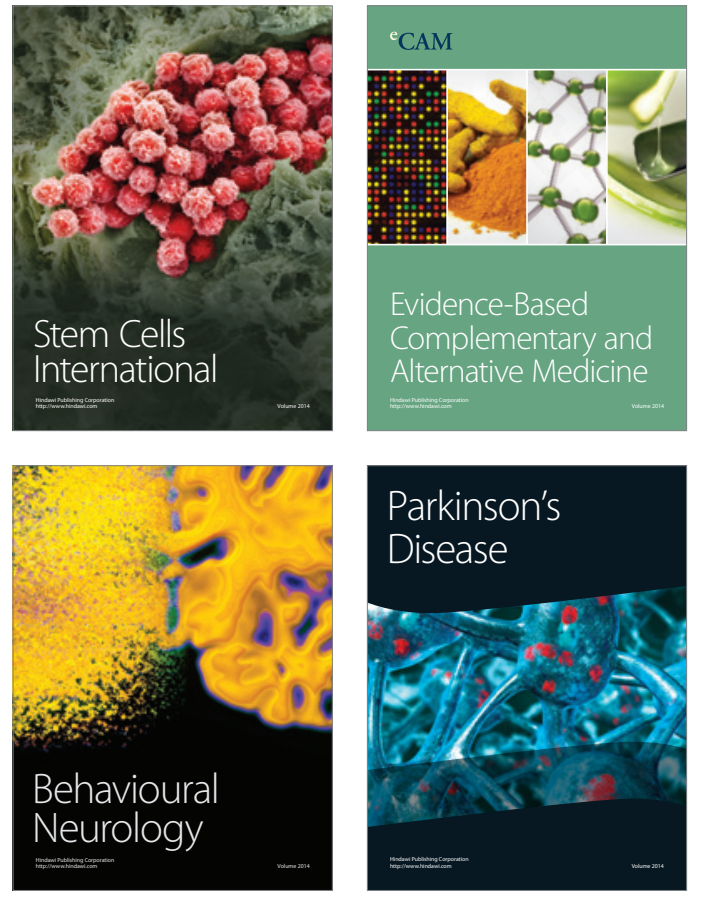
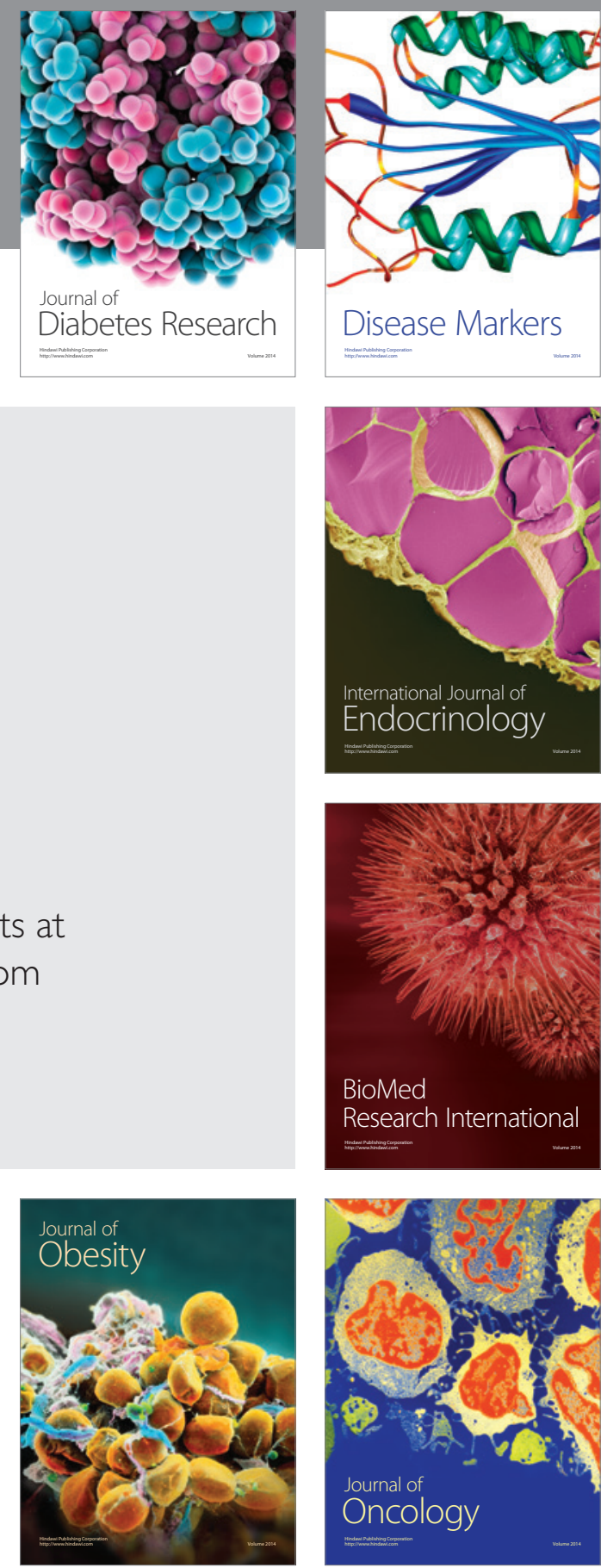

Disease Markers
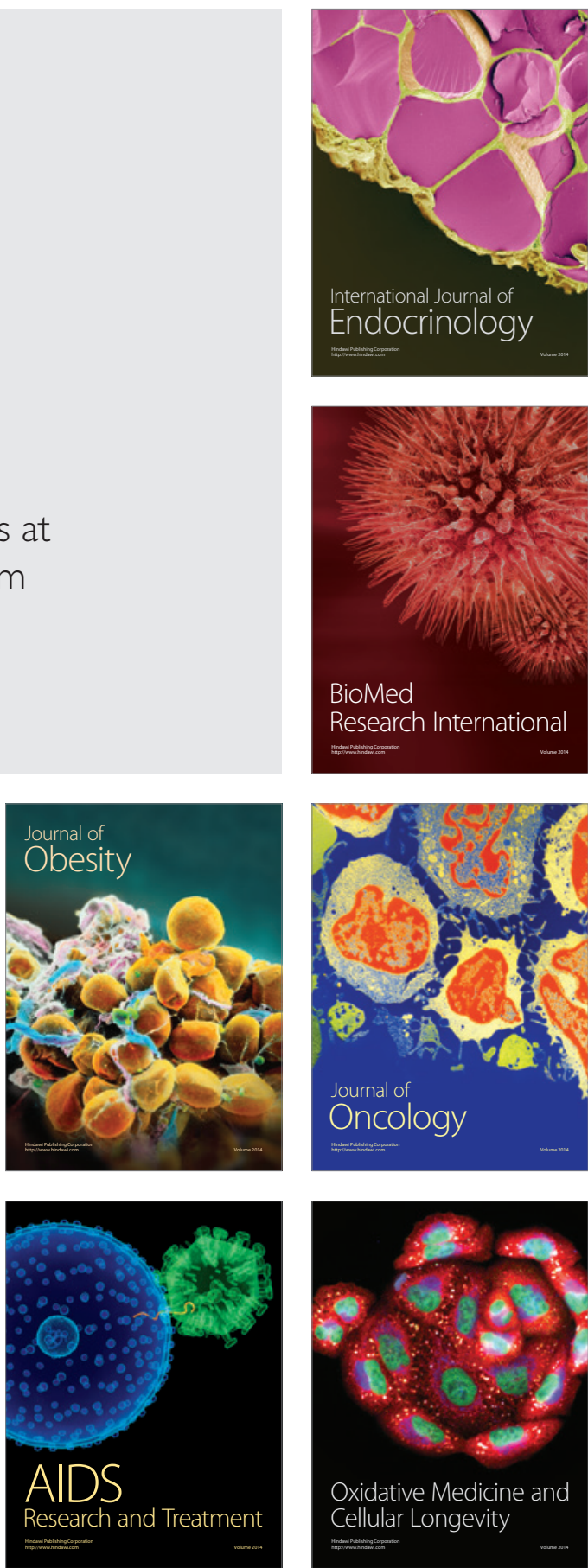